
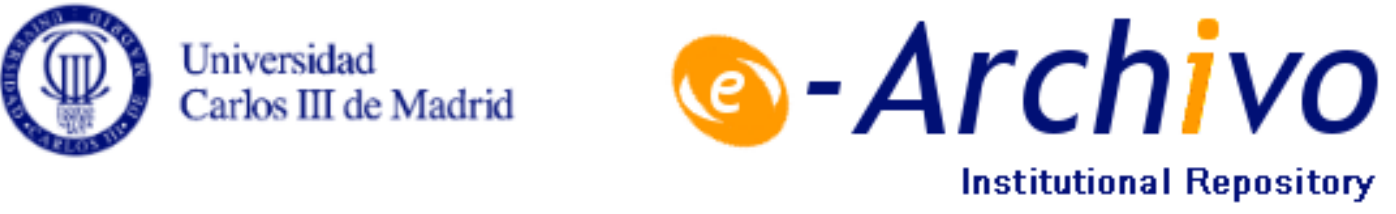

This document is published in:

Journal of Theoretical Biology 334 (2013) 7 October, pp. 35-44 DOI: 10.1016/j.jtbi.2013.06.004

(C) 2013 Elsevier

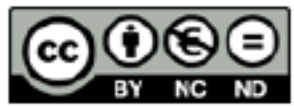

This work is licensed under a Creative Commons Attribution-NonCommercialNoDerivatives 4.0 International License. 


\title{
Degree of intervality of food webs: From body-size data to models
}

\author{
José A. Capitán ${ }^{\mathrm{a}, \mathrm{b}, \mathrm{c}, *}$, Alex Arenas ${ }^{\mathrm{b}, \mathrm{d}}$, Roger Guimerà e,f \\ ${ }^{a}$ Grupo Interdisciplinar de Sistemas Complejos (GISC), Spain \\ b Department d' Enginyeria Informàtica i Matemàtiques, Universitat Rovira i Virgili, 43007 Tarragona, Catalonia, Spain \\ ${ }^{\mathrm{c}}$ Centro de Astrobiología, INTA-CSIC, 28850 Torrejón de Ardoz, Madrid, Spain \\ ${ }^{\mathrm{d}}$ IPHES (COMPATHEVOL), Institut Catala de Paleoecologia Humana i Evolucio Social, Universitat Rovira $i$ Virgili, 43007 \\ Tarragona, Catalonia, Spain \\ ${ }^{\mathrm{e}}$ Institució Catalana de Recerca i Estudis Avançats (ICREA), 0810 Barcelona, Catalonia, Spain \\ ${ }^{\mathrm{f}}$ Department d' Enginyeria Química, Universitat Rovira i Virgili, 43007 Tarragona, Catalonia, Spain
}

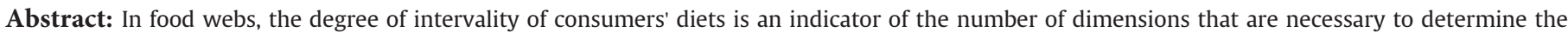

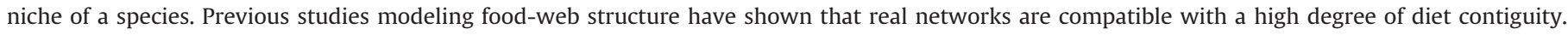

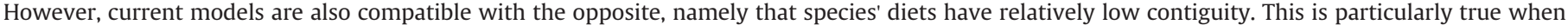

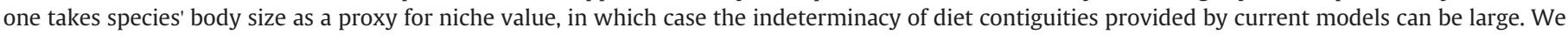

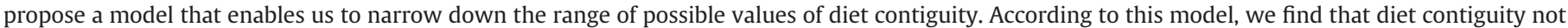
only can be high, but must be high when species are ranked in ascending order of body size.
\end{abstract}

Keywords: Diet contiguity, Ecological networks, Food-web structure, Niche dimension, Species size.

\section{Introduction}

Food webs are networks that describe trophic (consumerresource) interactions in communities (Cohen et al., 1990), and regularities in their structural properties are among the most prevalent in ecosystems (Camacho et al., 2002; Dunne et al., 2002; Cattin et al., 2004; Camacho and Arenas, 2005; Stouffer et al., 2005; Pascual and Dunne, 2006; Allesina et al., 2008). The existence of systematic patterns in food webs of very different origin and nature has encouraged researchers to propose models for their structure, with the aim of reproducing the observed patterns from simple food web assembly rules (Cohen and Newman, 1985;

* Corresponding author at: Centro de Astrobiología, INTA-CSIC, 28850 Torrejón de Ardoz, Madrid, Spain. Tel.: +34 915206425.

E-mail addresses: joseangel.capitan@cab.inta-csic.es (J.A. Capitán), alexandre.arenas@urv.cat (A. Arenas),roger.guimera@urv.cat (R. Guimerà).
Williams and Martínez, 2000; Cattin et al., 2004; Stouffer et al., 2006; Allesina et al., 2008; Capitán et al., 2009, 2011; Capitán and Cuesta, 2011). The design and evaluation of theoretical models for food-web structure is crucial to understand the persistence of ecological communities and their fragility against external perturbations (Stouffer et al., 2008, 2012; Capitán and Cuesta, 2010; Stouffer and Bascompte, 2011).

Theoretical models of food webs often rely on the concept of ecological niche. A species' niche was initially conceived as the set of relevant traits that determine the trophic position of a species in the network of trophic interactions (Hutchinson, 1957). The question of how many "niche dimensions" are relevant to represent species in their communities has given rise to a long debate in ecology (Cohen, 1977). It has been argued (Stouffer et al., 2006) that the structure of empirical food webs can be fairly well explained reducing the number of traits to simply one. If a single trait were enough to characterize the network of feeding interactions, species could be 
ordered in a way that each consumer had a contiguous diet, that is, each species would prey upon a set of consecutive resources. Hence consumer's diets could be represented as intervals in a onedimensional niche space. A food web in which all consumers' diets form continuous intervals along a single niche dimension is known as a perfectly interval food web. Non-interval webs, however, are networks such that no species ordering is possible for consumers' diets to be perfectly contiguous. The quasi-interval nature of real food webs has inspired the majority of recent models for food-web structure (Williams and Martínez, 2000; Stouffer et al., 2005; Allesina et al., 2008), but some researchers have pointed out that real networks are not perfectly interval and that models that generate perfectly interval food webs are therefore inappropriate (Cattin et al., 2004). Stouffer et al. (2006) solved the puzzle by demonstrating quantitatively that a small degree of diet non-contiguity is enough to generate networks whose intervality is compatible with that of empirical food webs. The small deviation from complete intervality suggests that a single trait or a small set of them are enough to capture the structure of feeding interactions and species' niches. Other studies have proven recently that the number of niche dimensions in food webs is low (Eklöf et al., 2013)—see, however, the work by Rossberg et al. (2010) regarding the dimensionality of the niche space.

Several candidates for the trait corresponding to the niche dimension were proposed in the past, most prominently body size (Lawton and Warren, 1988). Based on this correspondence between an "abstract" niche variable and body size, other models for foodweb structure have been proposed (Loeuille and Loreau, 2005; Lewis and Law, 2007; Petchey et al., 2008). Recent works (Guimerà et al., 2010; Stouffer et al., 2011; Zook et al., 2011) have dealt with the long-standing question of what determines the ecological niche of a species. These studies have tried to find an empirical property or a species trait playing the role of the niche dimension in models of food-web structure. As potential proxies for niche value, these works have considered species trophic position (measured in different ways) and body size (usually measured as the average, among a certain sample of individuals, of body masses or body lengths). Species in the network are ordered by the empirical property and the total number of gaps in the resulting diets is compared afterwards to a randomly ordered food web. All these works conclude that species body size explains the degree of intervality and the contiguity of diets in real food webs significantly better than random orderings or ranking species according to other quantities such as trophic positions.

In addition, species' size contributes to explaining the mesoscopic structure of ecological networks. Besides intervality, other topological properties such as the distribution of predators and prey (Camacho et al., 2002; Stouffer et al., 2005), or the presence of structural motifs in the network (Bascompte and Melián, 2005; Camacho et al., 2007; Stouffer et al., 2007), are relevant when it comes to characterizing the topology of natural food webs. In particular, the existence of compartments (i.e., groups of species that interact among themselves with higher probability than with species outside) has been put into correspondence with body size (Guimerà et al., 2010). The analysis of compartments in empirical ecological networks is best described using body size as a proxy for niche value. In accordance with previous related work, trophic level appears to be a poor proxy for niche value (Jennings et al., 2002; Woodward et al., 2005).

Here we show that, with existing food web models, one cannot in principle conclude that species' diets must be highly contiguous -high diet contiguity is compatible with observed data, but so is relatively low contiguity. This is particularly true when one takes body size as a proxy for niche value. To minimize this problem we propose a model that extends the generalized niche model (GNM) of Stouffer et al. (2006) by allowing predation on a certain range of resources whose niches are larger than the niche value of the consumer. The rationale behind this choice is supported by empirical evidence showing that consumer-resource body-size ratios in natural ecosystems are often smaller than one, i.e., consumer size is very often smaller than resource's size (Brose et al., 2006; Petchey et al., 2008). Our model describes several properties of real food webs better than previous models and, more importantly, enables us to narrow down the range of possible values of diet contiguity. Therefore, we show that diet contiguity, not only can be high, but must be high when species are ranked in ascending order of body size.

\section{Materials and methods}

\subsection{The generalized niche model}

The analysis of food-web intervality conducted by Stouffer et al. (2006) led them to generalize the niche model (NM) by Williams and Martínez (2000). The resulting generalized niche model (GNM) produces interaction networks with a certain number of gaps in consumer's diets. GNM food webs are generated as follows. Let $S$ be the number of species in the web. A niche value $n_{i}$ drawn from a uniform distribution in the interval $[0,1]$ is assigned to each species. Part of the diet of consumer $j$ is chosen as the subset of species that lie in the range $r_{j}=c x n_{j}$, where $x$ is drawn from a beta-distribution $f(x)=\beta(1-x)^{\beta-1}$ and $\beta=\left(S^{2} / 2 L\right)-1$, with $L$ being the total number of trophic interactions (links) in the network. The center $c_{j}$ of the range is chosen uniformly at random in the interval $\left[r_{j} / 2, n_{j}\right]$, and the parameter $c$ belongs to $[0,1]$. The rest of the diet consists of $\Delta k_{j}=(1-c) r_{j} S$ species chosen at random from those species $i$ not yet consumed by $j$ and whose niche values obey $n_{i} \leq n_{j}$.

The parameter $c$ measures diet contiguity. For $c=1$ one recovers the original perfectly interval NM with a one-dimensional niche space, since any species $i$ whose niche falls within the range $r_{j}$ is consumed by $j$. In contrast, for $c<1$ the diets of predators do not form a continuous interval. In the limit $c=0$ one recovers the generalized cascade model [GCM; Stouffer et al., 2005], since all prey $i$ of consumer $j$ are randomly chosen among the species for which $n_{i} \leq n_{j}$.

\subsection{Extending the generalized niche model}

We propose an extension of the generalized niche model (EGNM) that allows for a predator $j$ to have some of its noncontiguous prey with niche values larger than $n_{j}$ (upward consumption). To this end we introduce a new parameter $p$ in the model that tunes the maximum niche value $m_{j}$ for the noncontiguous prey of a consumer, so that species $j$ can consume from niches $n_{i} \leq m_{j}=n_{j}+p\left(1-n_{j}\right.$ ) (for $p=0$ we recover the GNM, and for $p=1$ any species is susceptible to be preyed upon). We call this parameter "probability of upward consumption". Our model proceeds as the GNM except for the random choice of the $\Delta k_{j}$ noncontiguous prey. If $n_{i} \leq n_{j}$, any prey $i$ (among those not already consumed in the contiguous part of the diet) can be selected with a uniform probability, as in the GNM. For $n_{j}<n_{i} \leq m_{j}$, however, we choose a (linear) decreasing probability to randomly select a prey (see Fig. 1). Any other decreasing function leads to similar results. This assumption reflects the fact that, indeed, for a small con-sumer, the larger the resource the smaller the likelihood to interact with it. The mathematical form of this distribution is

$$
f\left(n_{i}\right)= \begin{cases}\rho & \text { if } n_{i} \in\left[0, n_{j}\right] \backslash\left[c_{j}-r_{j} / 2, c_{j}+r_{j} / 2\right], \\ \rho\left(1-\frac{1}{p} \frac{n_{i}-n_{j}}{1-n_{j}}\right) & \text { if } n_{i} \in\left[n_{j}, n_{j}+p\left(1-n_{j}\right)\right], \\ 0 & \text { otherwise. }\end{cases}
$$



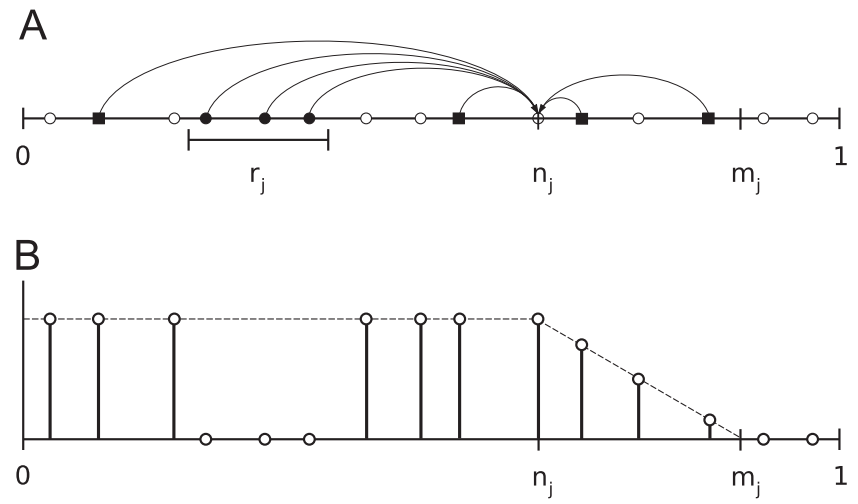

Fig. 1. (A) Scheme of the EGNM. Consumer $j$ is allowed to prey any species $i$ with $n_{i} \leq m_{j}=n_{j}+p\left(1-n_{j}\right)$. At $p=0$ we recover the GNM, and at $p=1$ any species can be consumed by $j$. We first draw the range $r_{j}$ of contiguous prey (black circles) for consumer $j$. Then we randomly choose $\Delta k_{j}=(1-c) r_{j} S$ prey (squares) in the interval $\left[0, m_{j}\right]$ according to the probability distribution depicted in (B), for which potential prey with $n_{i}>n_{j}$ are less likely to be selected.

Normalization imposes the condition $\sum_{n_{i}} f\left(n_{i}\right)=1$, where the sum is restricted to niche values not contained in $\left[c_{j}-r_{j} / 2, c_{j}+r_{j} / 2\right]$ or $\left[m_{j}, 1\right]$, which determines the normalization coefficient $\rho$. The random choice of species according to this discrete distribution continues until $\Delta k_{j}$ prey are assigned to the predator.

An interesting point is that, strictly speaking, the GNM is not compatible with some empirical food webs (Allesina et al., 2008). This is due to the fact that some of the non-contiguous prey observed in empirical food webs cannot be accounted for when randomly selected prey are restricted to have niche values smaller than the niche of the predator. Our EGNM model permits random consumption upwards in the niche axis, and therefore removes the constraint imposed on the niches values of non-contiguous prey. At least in the $p=1$ limit, and often much before that, our model is strictly compatible with any empirical food web.

\subsection{Diet contiguity estimation}

Estimating diet contiguity in empirical food webs is involved because niche values of species are unknown. To address this difficulty we assume, initially, that the niche values used in nichebased models of an empirical food web can be put into direct correspondence with the body size values reported for that network in the form of body mass or body length. As mentioned, this assumption is supported by recent work addressing how bodysize-ordered food webs compare to their random-ordered counterparts (Stouffer et al., 2011; Zook et al., 2011), as well as the analysis of compartments in empirical food webs (Guimerà et al., 2010). See Appendix A for a list of the empirical food webs used in this work and their main properties.

In particular, for each empirical food web, we order species from smallest to largest body size, thus yielding the ordering $P=\left\{s_{1}, s_{2}, \ldots, s_{S}\right\}$ where body sizes obey $w_{s_{1}}<w_{s_{2}}<\cdots<w_{s_{S}}$. The diet contiguity for such a permutation of species labels can be measured by the total number of species belonging to gaps in consumers' diets (Stouffer et al., 2006),

$G_{\mathrm{e}}=\mathcal{G}(P)=\sum_{i=1}^{S} \sum_{j=1}^{\gamma_{i}} g_{i j}$

where $\gamma_{i}$ stands for the number of gaps in the diet of species $i$ and $g_{i j}$ is the number of species present in the $j$-th gap. We list in Appendix A the empirical number of gaps $G_{e}$ yielded by the bodysize ordering for all the food webs with body-size data available.

\subsection{Validation metrics}

To compare the performance of both models when trying to reproduce the structure of real food webs, we have studied 12 statistical quantities plus the number of gaps in consumers' diets. Most of them are the usual descriptors of food webs (Williams and Martínez, 2000). Since our EGNM is in principle biased toward linking consumers to species with larger niche values, we have also focused in measures like the average number of loops, the average shortest path, or the average trophic level, which somehow should reflect this potential bias. The set of properties we have measured is:

1-3. Species types: the fraction $T$ of top (species with no predators), basal ( $B$, species with no prey) and intermediate $(I=1-B-T)$ species (Cohen et al., 1990).

4-5. The standard deviations of generality (GenSD) and vulnerability (VulSD). Normalized generality $\left(\Gamma_{i}\right)$ and vulnerability $\left(Y_{i}\right)$ of species $i$ are defined as Schoener (1989)

$\Gamma_{i}=\frac{1}{z} \sum_{j=1}^{S} a_{i j}, \quad r_{i}=\frac{1}{z} \sum_{j=1}^{S} a_{j i}$,

where $a_{i j}$ stand for the entries of the network's adjacency matrix $\left(a_{i j}=1\right.$ if species $i$ preys upon species $j$ and zero otherwise). The normalization with the linkage density $z=L /$ $S$ force mean $\Gamma_{i}$ and $r_{i}$ to equal unity, hence standard deviations can be compared across different webs.

6. Trophic similarity of a pair of species $\left(s_{i j}\right)$ measures the overlap of in- and out-going links in the web. It is the ratio between the number of common predators and prey and the total number of predators and prey (Martínez, 1991). For each web, the maximum similarity index of species $i$ is averaged over the network to obtain mean maximum similarity,

$\operatorname{MaxSm}=\frac{1}{S} \sum_{i=1}^{S} \max _{i \neq j} s_{i j}$

7. Average trophic level $(T L)$ : the trophic level $\ell_{i}$ of species $i$ has been computed as

$\ell_{i}=1+\frac{1}{a_{i}} \sum_{j=1}^{S} a_{i j} \ell_{j}$,

where $a_{i}=\sum_{j=1}^{S} a_{i j}$ is the number of prey in the diet of species $i$ (Levine, 1980). It equals 1 plus the weighted average of chain lengths from a species to a basal species, the weights being equal to $a_{i j} / a_{i}$ (i.e., each predator consumes equally from all its prey). Note that this quantity is inspired in the flow of energy from bottom to top occurring in the food web. The trophic position of each species is averaged across the web to yield the quantity $T L$.

8-9. Standard deviation of $\ell_{i}$ averaged across the web (TLSD) measures the degree of trophic specialization (Levine, 1980). Omnivory [Omniv, Polis, 1991] is the fraction of species that prey on different trophic levels (i.e., that are connected with basal species by food chains of different lengths).

10. Another measure of trophic position is the mean shortest path (Short) from each species to a basal species (Williams and Martínez, 2004).

11. The fraction of cannibals (self-consuming species, Cannib) quantifies the number of loops of length 1 .

12. The presence of non-trivial loops is measured by the average number of triangular loops (Loops). To calculate it, we use a matrix $(B)$ whose entries are $b_{i j}=a_{i j}-a_{i i}$ (i.e., the adjacency matrix without cannibalistic loops). The number of loops of length 3 starting from species $i$ can be obtained as the $i$-th diagonal element of the third power of $B$. We finally average this number across the web. 


\subsection{Maximum-likelihood parameter estimation}

Our validation metrics is formed by 13 statistical properties. As shown below, the observed number of gaps is normally distributed. We have also checked that the rest of statistical quantities follow Gaussian distributions when averaged over enough model realizations. For both the GNM and the EGNM we estimate model's log-likelihood as

$\log \mathcal{L}=-\sum_{i=1}^{k} \log \sigma_{i}-\frac{1}{2} \sum_{i=1}^{k} z_{i}^{2}$,

where the sum extends to the $k=13$ empirical properties $X_{i}$ that we have measured for each food web, and $z_{i}=\left(\left\langle X_{i}\right\rangle-X_{i, \mathrm{e}}\right) / \sigma_{i}$ is the corresponding $z$-score, $\left\langle X_{i}\right\rangle$ and $\sigma_{i}^{2}$ being the model average and variance of $X_{i}$, respectively, and $X_{i, \mathrm{e}}$ the observed value of that property in empirical networks. Mean and variance will be certain functions of model parameters, so the likelihood function will depend implicitly on model parameters-on $c$ for the GNM, or on $(c, p)$ for the EGNM. Model parameters will be estimated maximizing the log-likelihood function. Note that Eq. (6) assumes that all the statistical properties are independent, which is not necessarily true. For example, the fraction of top, intermediate and bottom species are correlated since their sum is equal to one. Ignoring one of these three variables, however, leads to comparable estimates. For the sake of simplicity, we assume that correlations between different quantities are weak and use Eq. (6) as model's log-likelihood estimate.

Our maximum likelihood (ML) estimation procedure focuses of network properties of ecological interest. We have not used the likelihood function for the GNM (Allesina et al., 2008) and its counterpart for the EGNM because, in practice, due to inevitable mismatches between statistical models and reality, a likelihood approach could end up concentrating on features of the data that are actually not biologically interesting. However, a propertybased approach (Kendall et al., 1999; Reuman et al., 2006) focuses on statistical properties of ecological interest. In our case, properties like the number of gaps in consumers' diets, or those properties that can be altered by permitting upward consumption (such as Loops, Short, TL, TLSD, Omniv, etc.), are relevant for our purposes and have been taken into account explicitly in our estimation of log-likelihood.

Model selection follows the Akaike Information Criterion (AIC). For each model, we have calculated the index

$A I C=2 n-2 \log \mathcal{L}_{\max }$,

given the ML parameter estimates, $n$ being the number of parameters of the model. By choosing the model with minimum AIC index we are minimizing the information loss among all candidate models (Akaike, 1974).

\section{Results}

\subsection{Diet contiguity confidence intervals}

In their work, Stouffer et al. (2006) were interested in determining the maximum amount of diet contiguity $c$ compatible with the number of gaps observed in empirical food webs. Accordingly, they used the ordering of species that minimizes Eq. (2) for each empirical food web and took the maximum value of $c$ compatible with empirical observations. However, realizations of the GNM with different values of the diet contiguity allow for the calculation of the full 95\% confidence intervals of $c$. To obtain the confidence intervals one calculates the cumulative probability $\operatorname{Pr}\left(G \leq G_{e} ; c\right)$ to observe a number of gaps $G$ smaller than the empirical value $G_{\mathrm{e}}$ as a function of prey contiguity $c$. Such probability can be easily calculated because we can not reject the null hypothesis that the number of gaps is normally distributed (Fig. 2a). The intervals are indeed quite broad for body-size orderings (Fig. 3, first panel).

Additionally, under the assumption that body size corresponds to niche value, the empirical intervality is, in general, smaller than the maximum possible (Zook et al., 2011), and the confidence interval grows (Fig. 2). Compared to the GNM, our EGNM introduces more gaps in consumers' diets, both because it allows for larger number of gaps and because, in general, gaps are larger. This fact can be observed in Fig. 2b, and we discuss its implications below.

We use a collection of food webs for which body-size data are available. Reported size data refer either to average body masses or average body lengths of individuals (Brose et al., 2005), so we generically use the term 'body size' to encompass both cases. For each food web we determine the empirical number of diet gaps $G_{e}$ derived from the body-size ordering (see Appendix A for a list of values and details about these orderings). Then we generate stochastic realizations of our EGNM for different values of the probability $p$ of upward consumption, i.e., the parameter that determines to what extent a species can have non-contiguous prey with niche values higher than itself $(p \in[0,1])$. For each $p$, averages over realizations lead to the cumulative probability $\operatorname{Pr}\left(G \leq G_{e} ; c\right)$. Fig. 3 shows an example of such histograms for the set of empirical values $\left(S, z\right.$ and $G_{\mathrm{e}}$ ) that correspond to the Benguela marine ecosystem (Yodzis, 1998).
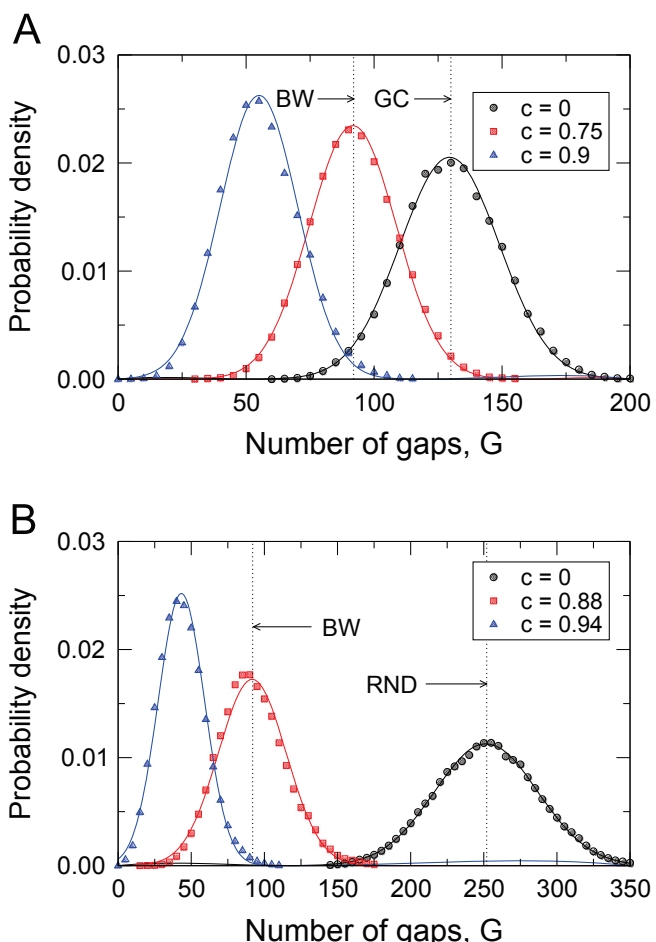

Fig. 2. Diet contiguity estimation for the Benguela food web. (A) Histogram of the number of gaps [cf. Eq. (2)] obtained for the GNM $\left(10^{5}\right.$ model realizations) at three different values of diet contiguity $c$. The hypothesis that $G$ is a normally distributed variable can not be rejected (continuous lines). The average number of gaps observed at $c=0$ (GCM, 130 gaps) and the empirical number of gaps (body size ranking, $G_{e}=92$ ) are marked with vertical lines. (B) Histograms of the number of gaps obtained for a probability of upward consumption $p=1$ and three values of prey contiguity $c\left(10^{5}\right.$ EGNM realizations). Species number $S$ and linkage density $z$ are chosen to match Benguela values. Histograms do not depart from normal distributions (continuous lines) in a significant manner. The largest number of gaps (252 at $c=0$, marked as RND) is far larger than the empirical number of gaps $\left(G_{\mathrm{e}}=92\right.$, body-size ordering, marked as BW). The distribution for $c=0.88$ (whose average matches the empirical value) scarcely overlaps with its completely random counterpart for $c=0-$ compare with $(\mathrm{A})$. 

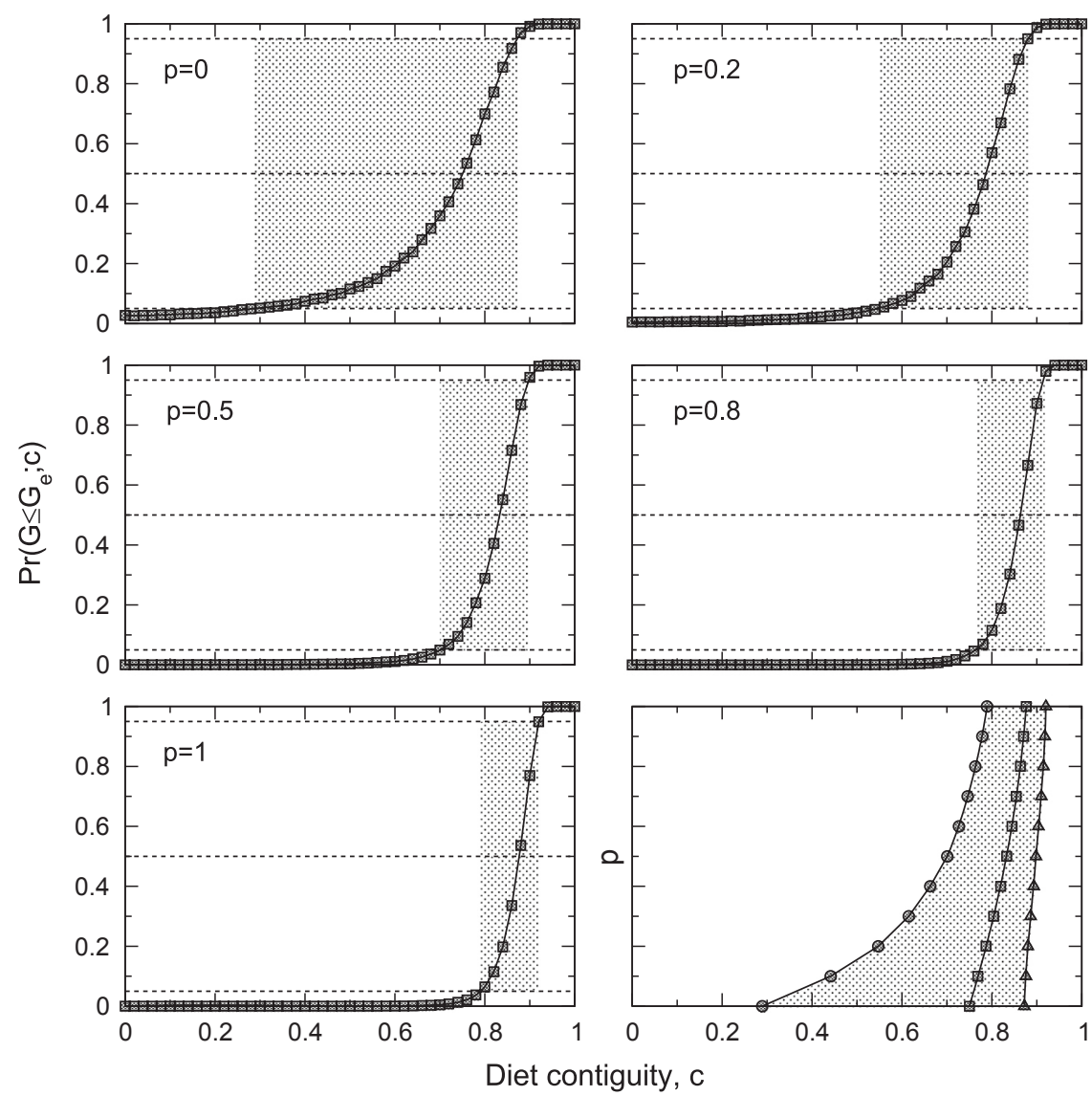

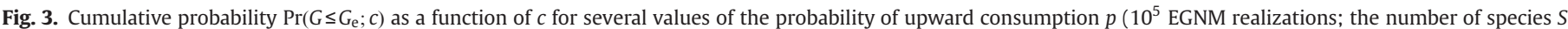

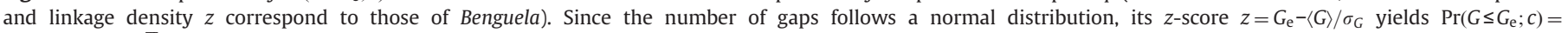

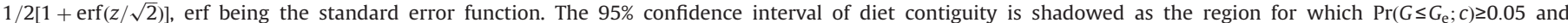

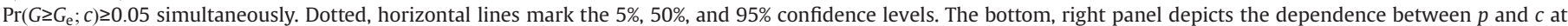

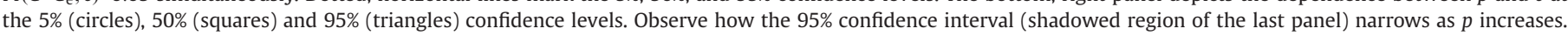

Increasing the probability of upward consumption leads to narrower 95\% confidence intervals for prey contiguity $c$ because larger values of $p$ imply the possibility of more and larger gaps, so the small number of gaps observed in real networks become compatible only with high diet contiguity (see Fig. 4). The narrowing of the range of possible values for the diet contiguity $c$ is important. Indeed, Stouffer et al. (2006) showed that a small deviation from perfect contiguity $c \leqslant 1$ is enough to account for the gaps observed in real food webs, but one may argue that very low diet contiguity in the GNM is also compatible with the data. For example, at a $5 \%$ confidence level, $c=0.289$ is also compatible with the number of gaps observed in Benguela (Fig. 3, panel $p=0$ ). The GNM is therefore compatible both with high and low values of diet contiguity. In contrast, when species are allowed to have noncontiguous prey anywhere in the niche space $(p=1), c$ must be confined to the interval $(0.789,0.920)$ for the same food web. Therefore, assuming that our EGNM at least as accurate as the GNM (we will focus on this assumption in the model selection section), since the confidence interval for diet contiguity narrows and moves towards values closer to 1 , there is evidence that not only diet contiguity could be high, as the GNM model itself predicts, but that it must be so.

\subsection{Model selection and parameter estimates}

ML parameter estimation has been performed by averaging each statistical property over 5000 model realizations except for Caribbean Reef, Caricaie Lakes (2000 realizations), and Weddell (500 realizations), the largest food webs in the dataset. We have measured the log-likelihood on a $101 \times 101$ grid of equally spaced pairs $(c, p) \in[0,1] \times[0,1]$. ML parameter estimates are listed in Table 1. Moreover, Table 1 shows the AIC-index difference between the EGNM and the GNM, $\Delta=A I C-A I C_{0}$. If $\Delta<0$, the EGNM is expected to perform better than the GNM. Our model is selected in 13 out of 17 empirical food webs, which means that the additional parameter we introduce produces an information loss significantly smaller than the GNM.

Note that using the explicit body-size ordering moves diets away from perfect intervality, which in turn disfavors the proximity to 1 of our estimation of prey contiguity. Despite this fact, the c-estimates listed in Table 1 are rather close to 1 in most cases. Therefore, the introduction of a new parameter controlling upward interactions in the niche axis enables us to narrow down the range of possible values of prey contiguity $c$ and provides further evidence that $c$ must indeed be close to 1 .

Once one of the models has been selected, the relative likelihood $e^{-\Delta / 2}$ can be interpreted as the relative probability that the other model minimizes the (estimated) information loss. We list relative likelihoods in Table 1. Observe that the GNM is selected in 4 cases, but even for those webs the relative likelihood that the EGNM minimizes the information loss is never negligible-it is always larger than 0.44. Conversely, when our model is chosen, the relative likelihood is smaller than 0.21 , being negligible in most cases.

ML parameter estimates for diet contiguity and probability of upward consumption have been plotted together with the 95\% confidence intervals for diet contiguity as a function of the 


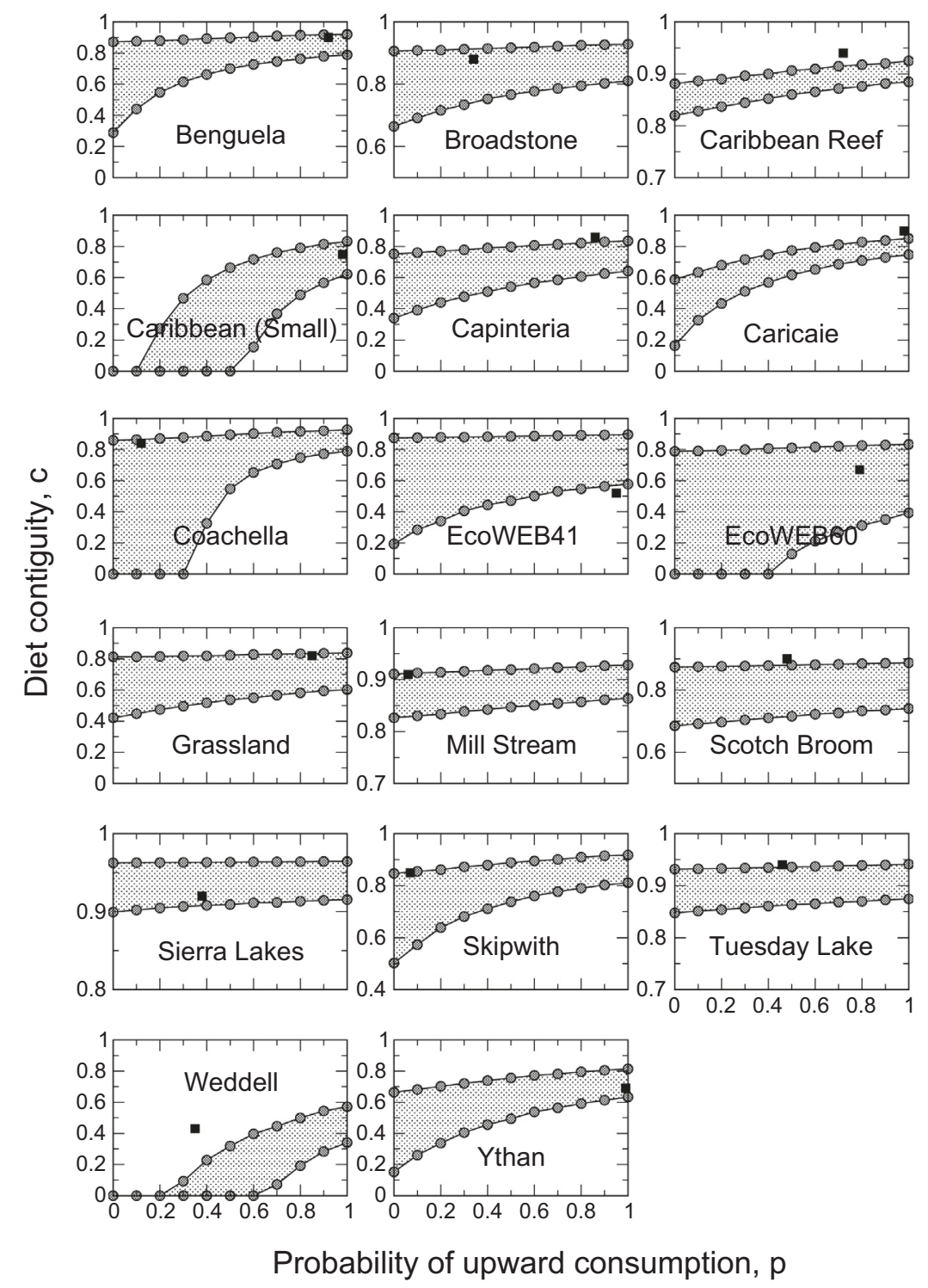

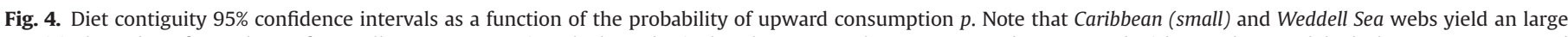

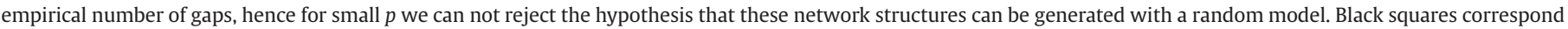
to the $(p, c)$ pairs yielded by ML estimation.

probability of upward consumption in Fig. 4. Except for EcoWEB41, all the parameter estimates lie within the interval determined for the corresponding value of $p$ or are above-but close to-the maximum diet contiguity compatible with the data at a $95 \%$ confidence level.

Fig. 5 summarizes the performance of the EGNM for each statistical property separately. Using the ML estimated parameters, we calculate the $z$-score of each quantity from $10^{5}$ model realizations. The model that renders the $z$-score $z_{i}$ closest to zero will explain better property $i$. In terms of the percentage of properties that are better described, EGNM's success ratio equals 58\% - out of a total of 221 possibilities (17 food webs evaluated against 13 properties), 128 were better explained by our EGNM.

We observe a tendency for EGNM to capture some properties better than the GNM, in particular the number of gaps in predator's diets and the fraction of cannibals, as well as the quantities that measure species' trophic position (TL, TLSD, and Short). Note that EGNM's potential bias toward upward consumption in the niche axis increases trophic-level values and shortestpath lengths. Interestingly, this bias additionally explains why the standard deviation of generality (GenSD) is better accounted for by the EGNM, since fluctuations in the number of prey of each predator will be larger when $p \approx 1$. On the other hand, although properties like the fraction of loops and omnivory are influenced by EGNM's bias toward upward consumption, they are reasonably well represented by our model.

\section{Discussion}

Food web intervality has long been studied as a proxy for the structure of the niche space, which determines the structure of food webs in ecosystems. However, it was only recently that Stouffer et al. (2006) provided a way to quantify, not whether a food web is interval or not, but to what extent the network is interval. This quantification is challenging in two respects. First, the quantification of diet contiguity depends on the model one uses to generate null food webs. Second, to determine the degree of intervality of a food web one needs to order species according to their niche "values," which are unknown a priori.

With regard to the first challenge, we argue that with existing models low diet contiguity is also compatible with the degree of 
Table 1

Parameter estimates of ML optimization for the GNM $\left(c_{0}\right)$ and the EGNM $(c, p)$. Differences between GNM and EGNM $\log$-likelihoods $\left(\log \mathcal{L}_{0}\right.$ and $\log \mathcal{L}$, respectively) are provided, as well as the difference $\Delta=A I C-A I C_{0}$ of Akaike information coefficients. The relative likelihood $e^{-\Delta / 2}$ has been listed for each empirical food web. When the GNM is selected, relative likelihoods have been marked in boldface

\begin{tabular}{llllllll}
\hline Food web & $c$ & $p$ & $c_{0}$ & $\log \left(\mathcal{L} / \mathcal{L}_{0}\right)$ & $\Delta$ & $e^{-\Delta / 2}$ & $\begin{array}{l}\text { Selected } \\
\text { model }\end{array}$ \\
& & & & & & & \\
\hline Benguela & 0.90 & 0.92 & 0.85 & 0.72 & 0.56 & $\mathbf{0 . 7 6}$ & GNM \\
Broadstone & 0.88 & 0.34 & 0.88 & 2.92 & -3.84 & 0.15 & EGNM \\
stream & & & & & & & \\
Caribbean reef & 0.94 & 0.72 & 0.94 & 36.6 & -71.1 & $<10^{-15}$ & EGNM \\
Caribbean & 0.75 & 0.98 & 0.82 & 56.2 & -110.4 & $<10^{-23}$ & EGNM \\
(small) & & & & & & & \\
Caricaie lakes & 0.90 & 0.98 & 0.91 & 133.6 & -265.3 & $<10^{-57}$ & EGNM \\
Carpinteria & 0.86 & 0.86 & 0.83 & 0.18 & 1.64 & $\mathbf{0 . 4 4}$ & GNM \\
Coachella & 0.84 & 0.12 & 0.85 & 0.71 & 0.58 & $\mathbf{0 . 7 5}$ & GNM \\
EcoWEB41 & 0.52 & 0.95 & 0.90 & 22.6 & -43.2 & $<10^{-9}$ & EGNM \\
EcoWEB60 & 0.67 & 0.79 & 0.81 & 7.95 & -13.9 & $<10^{-3}$ & EGNM \\
Grassland & 0.82 & 0.85 & 0.81 & 0.70 & 0.61 & $\mathbf{0 . 7 4}$ & GNM \\
Mill stream & 0.91 & 0.06 & 0.92 & 3.42 & -4.84 & 0.09 & EGNM \\
Scotch broom & 0.90 & 0.48 & 0.92 & 4.24 & -6.48 & 0.04 & EGNM \\
Sierra lakes & 0.92 & 0.38 & 0.94 & 2.54 & -3.08 & 0.21 & EGNM \\
Skipwith pond & 0.85 & 0.07 & 0.88 & 4.95 & -7.90 & 0.02 & EGNM \\
Tuesday lake & 0.94 & 0.46 & 0.92 & 7.34 & -12.7 & $<10^{-2}$ & EGNM \\
Weddell & 0.43 & 0.35 & 0.55 & 122.0 & -241.9 & $<10^{-52}$ & EGNM \\
Ythan & 0.69 & 0.99 & 0.82 & 100.5 & -198.9 & $<10^{-43}$ & EGNM \\
& & & & & & & \\
\hline
\end{tabular}

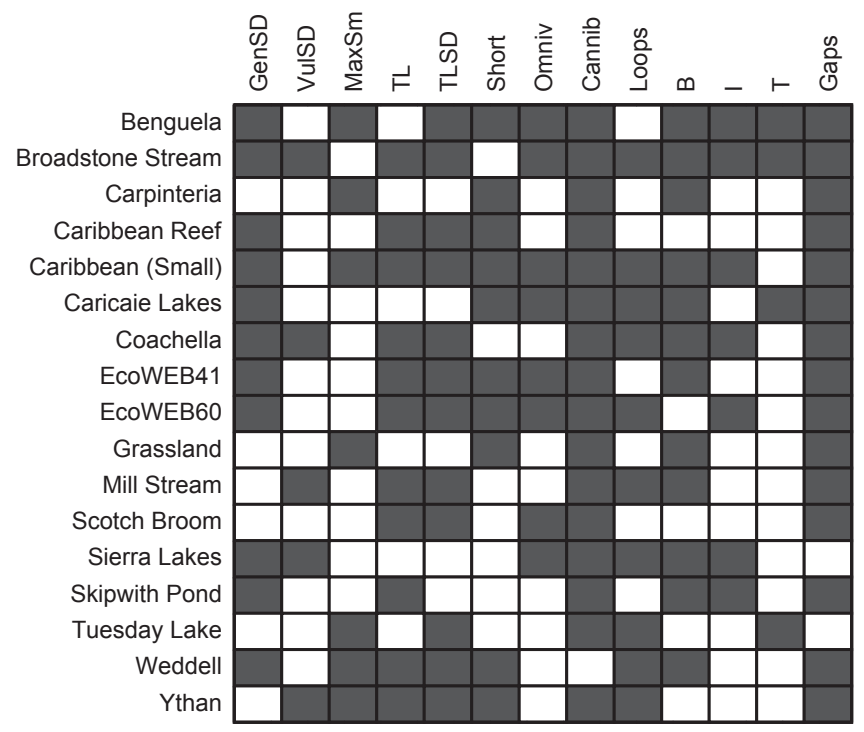

Fig. 5. For each food web, we mark with a full, black box the statistical properties that are better represented for EGNM webs with a non-zero probability of upward consumption. Blank boxes denote that the GNM captures better the empirical property.

intervality observed in real food webs. Therefore, we propose a model whose intervality, as defined in Stouffer et al. (2006), i s much more sensitive to the parameter that controls diet contiguity. The situation is conceptually similar to trying to estimate the value of a hidden variable in a system-one should always choose a proxy that is very sensitive to the value of the hidden variable. Importantly, the proxy should not be very sensitive to other variables, but only to the one we are interested in. In the context of food webs, this means that we are concerned with models whose intervality changes quickly with diet contiguity while other network properties remain close to those observed empirically. Our extension of the generalized niche model fulfills these requirements-as we show in Appendix B, when the intervality of model webs stops being compatible with empirically observed values, other network properties are still closer to their empirical values than for the GNM averages.

With regard to the second challenge, multiple works have pointed out that body size is at least a reasonable proxy for niche value, and certainly the best proxy we have been able to find. Zook et al. (2011) have compared the degree of intervality of empirical food webs when trophic positions are chosen as proxy for niche values. Although they conclude that body size can not completely explain observed patterns in food webs, it outperforms the results when compared to trophic levels (measured in three different ways). Therefore, we argue that ordering species by body size should yield reasonably good estimates of diet contiguity. In practice, however, using body size broadens the range of estimates of diet contiguity and biases their value down. Even then, our more sensitive model enables us to conclude that intervality must be high.

Our EGNM produces food webs that are consistent with empirical data when body size is chosen as a proxy for niche values. In particular, our model solves the inconsistency of the GNM pointed out by Allesina et al. (2008), who found some instances for which GNM-based webs could not reproduce the observed maximum number of non-contiguous prey per predator. This drawback is inherent to the GNM, which restricts the random assignment of non-contiguous prey downwards in the niche axis. Our extension relaxes this constraint and permits species orderings that are compatible with the interaction patterns observed in empirical food webs.

Despite the acknowledged relevance of parasitic interactions in food webs (Lafferty et al., 2006), we have focused in this work on networks that are almost free of them. We have treated as equivalent both predatory and parasitic interactions when parasites or parasitoids are present in the data (for example, in Scotch Broom and Grassland webs). Although parasite-parasitoid interactions are weakly dependent on body size, it could be relevant to carry out a similar study for more realistic data that incorporates parasitism in greater proportions (Lafferty et al., 2006).

The analysis performed by Stouffer et al. (2011) reveals to what extent body size is a significant explanatory variable accounting for a single niche dimension, and this degree of significance strongly depends on the evolutionary history of species. It seems that other latent traits are also responsible for species' roles in food webs. Importantly, it has been demonstrated that closely related species, in terms of their phylogeny, exhibit similar niches. The interplay between phylogenetic and ecological factors may be crucial in the determination of species' niche values distribution within a single trophic network (Rezende et al., 2009). The incorporation of the empirical, probably heterogeneous distribution of species over the niche space to mechanistic models of food-web structure reveals itself as a key question to address in the future.

\section{Acknowledgments}

We thank D.B. Stouffer for valuable comments and suggestions. J.A.C. acknowledges the support of a Juan de la Cierva Postdoctoral Fellowship. This work was supported by a James S. McDonnell Foundation Research Award (R.G.), European Union Grant PIRG-GA2010-277166 (R.G.), Spanish Ministerio de Ciencia e Innovación (MICINN) Grants FIS2009-13370-C02-01 (A.A.), FIS2010-18639 (R.G.), PRODIEVO, and FIS2011-27569 (J.A.C.), Comunidad de Madrid Grant MODELICO-CM (J.A.C.) and by Generalitat de Catalunya 2009-SGR838 (A.A.). 
Table A1

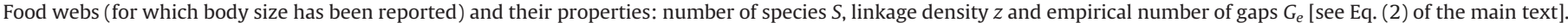

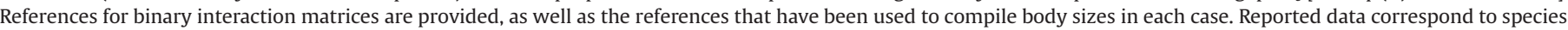
body length for Grassland, Mill Stream, Scotch Broom, Sierra Lakes, Skipwith Pond and Weddell Sea, and to species body mass for the remaining webs.

\begin{tabular}{|c|c|c|c|c|c|}
\hline Food web & Adjacency matrix reference & Body-size reference & $S$ & $z$ & $G_{\mathrm{e}}$ \\
\hline Benguela & Yodzis (1998) & Yodzis (1998) & 29 & 7.0 & 92 \\
\hline Broadstone stream & Woodward et al. (2005) & Brose et al. (2005) & 28 & 5.6 & 67 \\
\hline Caribbean Reef & Bascompte et al. (2005) & Bascompte et al. (2005) & 207 & 9.8 & 6969 \\
\hline Caribbean (small) & Opitz (1996) & Reide (unpublished) & 50 & 11.1 & 606 \\
\hline Caricaie Lakes & Cattin Blandenier (2004) & Brose et al. (2005) & 135 & 10.0 & 4640 \\
\hline Carpinteria & Lafferty et al. (2006) & Lafferty et al. (2006) & 72 & 3.3 & 705 \\
\hline Coachella & Polis (1991) & Reide (unpublished) & 26 & 8.8 & 75 \\
\hline EcoWEB41 & Cohen (1989) & Jonsson (1998) Jonsson & 19 & 2.7 & 27 \\
\hline EcoWEB60 & Cohen (1989) & $(1998)$ & 33 & 2.1 & 114 \\
\hline Grassland & Dawah et al. (1995) & Brose et al. (2005) & 55 & 1.6 & 246 \\
\hline Mill stream & Ledger, Edwards, and Woodward (unpublished) & Brose et al. (2005) & 74 & 5.0 & 483 \\
\hline Scotch broom & Memmott et al. (2000) & Brose et al. (2005) & 47 & 2.0 & 147 \\
\hline Sierra lakes & Harper-Smith et al. (2005) & Brose et al. (2005) & 32 & 6.0 & 36 \\
\hline Skipwith pond & Warren (1989) & Brose et al. (2005) & 34 & 7.6 & 135 \\
\hline Tuesday lake & Cohen et al. (2009) & Cohen et al. (2009) & 51 & 4.7 & 170 \\
\hline Weddell & Jacob, Brey, and Mintenbeck (unpublished) Hall & Brose et al. (2005) & 440 & 4.2 & 53,088 \\
\hline Ythan & and Raffaelli (1991) & Cohen et al. (2009) & 92 & 4.5 & 1594 \\
\hline
\end{tabular}

\section{Appendix A. Dataset description}

We use a collection of ecological networks for which body size (in the form of averaged body masses or averaged body lengths of sampled individuals) has been measured and reported. Table A1 lists, for each food web, the reference which contains the network of (binary) interactions, the reference used to compile body-size data, the number of species $S$ of the food web, the linkage density $z$, and the empirical number of gaps $G_{e}$ obtained when species are ranked in ascending order of body sizes.

The correspondence between species identity and body size was not unambiguous, i.e., for most of the webs there were two or more species recorded to have the same body size. Note that any ordering in each group of equivalent species is compatible with the ranking in ascending order of sizes. In most of the cases we can enumerate all the possible permutations of equivalent species. Therefore we choose the ordering that minimizes the number of gaps among all the permutations of these subsets. There were only two webs (Grassland and Weddell Sea) for which the exhaustive enumeration of all possible permutations of each degenerate subset was numerically out of the reach. In those cases, we randomly sample the possible orderings by transposing pairs of species within the same degenerate subset, and choose the minimum number of gaps after $10^{6}$ species transpositions. We have checked that the variability introduced by this degeneracy do not change dramatically the empirical number of gaps (i.e., we have calculated the maximum number of gaps compatible with the reported sizes and turns out to be comparable with the minimum value).

Body sizes have been compiled using different sources (see Table A1). In some cases we used data provided as online supporting material in several publications. That was the case of Caribbean Reef (Bascompte et al., 2005), Tuesday Lake ${ }^{1}$ and Ythan Estuary (Cohen et al., 2009). Most of sizes were retrieved from the article by Brose et al. (2005), which constitutes a compilation of consumer-resource interactions including either average body mass or average body length for different consumer-resource pairs. Most of the interactions listed in Brose et al. (2005) were extracted for previously published food webs. In many cases, the food web reported by Brose et al. (2005) does not coincide with the web reported in the original article. For example, although Benguela is one of the food webs provided by Brose et al.

${ }^{1}$ Cohen et al. (2009) provided samples of Tuesday Lake performed in 1984 and 1986. Here we chose the 1986 sample.
(2005), it has some missing links when compared with the original reference (Yodzis, 1998). We have used the database by Brose et al. (2005) to extract the connectivity structure for Broadstone Stream, Caricaie Lakes, ${ }^{2}$ Grassland, Mill Stream, Scotch Broom, Sierra Lakes, Skipwith Pond, a n d Weddell Sea, although the number of species and linkage density slightly differed from those originally reported.

On the other hand, the text data file provided by Brose et al. (2005) specifies the life stage of each species in each consumerresource interaction. We have ignored life stages because taking them into account does not introduce any difference in the resulting network, except for Broadstone Stream, Caricaie Lakes and Skipwith Pond. For these three webs, life stage differences exclusively appear for the same species acting either as consumer (life stage is recorded as, say, adult) or resource (life stage is recorded as juvenile, for instance). Therefore, if different life stages are considered as different nodes-as in Petchey et al. (2008)-resource life stages will be always regarded as basal species, which obviously introduces an artifact in the resulting web. Moreover, although species can exhibit different life stages, reported body sizes are exactly the same irrespective of the life stage. Therefore we have omitted life stage differences when constructing the networks. In those three webs, the differences introduced by ignoring life stages in the number of species and linkage density are small. In particular, ignoring life stage in Skipwith Pond yields a food web with the same taxa as reported in the original reference (Warren, 1989).

We have observed two additional issues when processing the data files to extract each food web:

1. Brose et al. (2005) report links for Scotch Broom that form a disconnected graph. We have maintained the giant (weakly) connected component since empirical data are to be compared with model realizations, which yield connected networks. We have checked that the remaining food webs form connected graphs.

2. We have debugged the text file provided by Brose et al. (2005) because sometimes species names contained blank spaces. Automatic text processing can mistakenly recognize as different those taxa which appear simultaneously with and without blank spaces.

\footnotetext{
${ }^{2}$ Brose et al. (2005) report data of 8 samplings of Caricaie Lakes in different geographical locations. We chose the sample of a triennially mown vegetation dominated by Orchio-Schoenetum nigricantis (Cattin Blandenier, 2004).
} 
Table A2

Values of model parameter $c$ (diet contiguity) for the EGNM and the GNM $\left(c_{0}\right)$ at a $5 \%$ confidence level in the number of gaps. At that level, empirical values of the number of gaps stop being compatible with model averages. The probability of upward consumption $p$ has been chosen as in the main text ( $p=0$ for the GNM; see Table 1 of the main text for EGNM's estimates). Observe that, in some instances, diet contiguities are zero. In such cases, the empirical number of gaps takes values that render these webs indistinguishable from their random counterparts, see Fig. 4 of the main text and Stouffer et al. (2006).

\begin{tabular}{lll}
\hline Food web & $c$ & $c_{0}$ \\
\hline Benguela & 0.78 & 0.29 \\
Broadstone stream & 0.74 & 0.66 \\
Caribbean reef & 0.87 & 0.82 \\
Caribbean (small) & 0.61 & 0.00 \\
Caricaie lakes & 0.74 & 0.16 \\
Carpinteria & 0.65 & 0.45 \\
Coachella & 0.00 & 0.00 \\
EcoWEB41 & 0.57 & 0.19 \\
EcoWEB60 & 0.31 & 0.00 \\
Grassland & 0.59 & 0.42 \\
Mill stream & 0.83 & 0.83 \\
Scotch broom & 0.71 & 0.69 \\
Sierra lakes & 0.91 & 0.90 \\
Skipwith pond & 0.55 & 0.50 \\
Tuesday lake & 0.86 & 0.85 \\
Weddell & 0.00 & 0.00 \\
Ythan & 0.63 & 0.15 \\
\hline
\end{tabular}

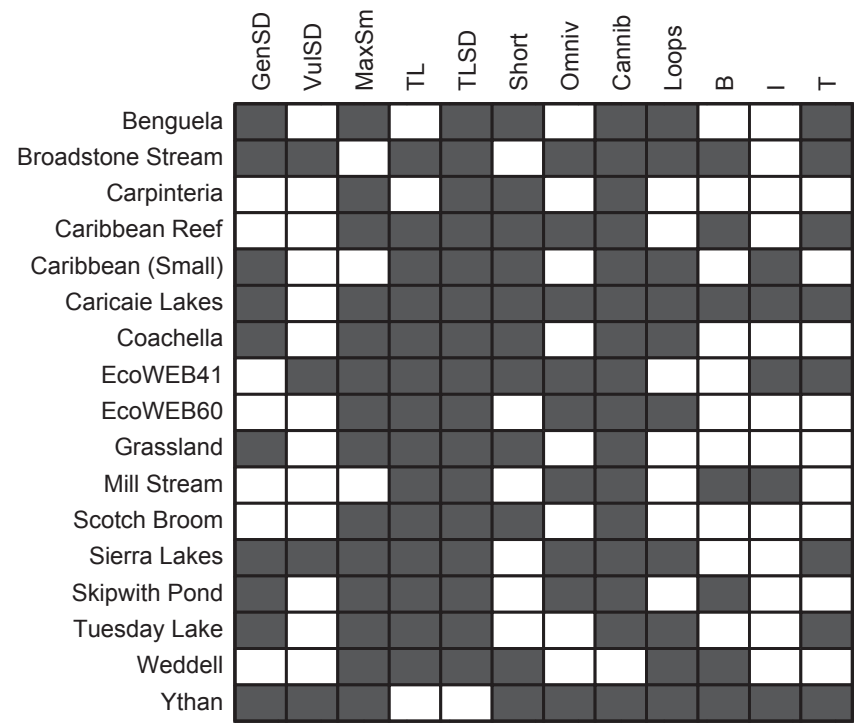

Fig. B1. Statistical descriptors for each food web in the dataset. Diet contiguities in model webs correspond to a $5 \%$ confidence level in the number of gaps. Success ratio is equal to $61 \%$. Color code is the same as in Fig. 5 of the main text. (For interpretation of the references to color in this figure caption, the reader is referred to the web version of this article.)

\section{Appendix B. Model performance at fixed confidence level for intervality}

To check that EGNM's intervality changes quickly with diet contiguity $c$ while other statistical properties remain close to their empirical values, we have determined the values of $c$ at a $5 \%$ confidence level, both for the EGNM and the GNM, for all food webs in the collection, keeping fixed the probability of upward predation estimated with maximum likelihood (see Table 1 of the main text and recall that $p=0$ for the GNM). At that confidence level, EGNM and GNM are expected to perform worse. Indeed, for these values of $c$ (which have been listed in Table A2), the number of gaps obtained for model networks start to be incompatible with empirical intervalities. We look at the remaining statistical properties and compare the EGNM and GNM values in order to determine which model yields quantities closer to the empirical values.

Results have been reported in Fig. B1. We have averaged up to $10^{5}$ model realizations and obtained the corresponding $z$-scores for all statistical properties except the number of gaps. Black boxes mean that the EGNM $z$-score is closest to zero than the GNM $z$-score, and white otherwise. We measure overall model's performance as the fraction of properties that EGNM closely describes when compared to GNM. Success ratio is equal to $61 \%$ in this case. This implies that our model is very sensitive to the parameter that controls diet contiguity, in the sense that the remaining statistical properties remain close to the empirical values whereas intervality has changed abruptly, far from being close to the empirically observed values.

\section{References}

Akaike, H., 1974. A new look at the statistical model identification. IEEE Trans. Autom. Control 19, 716-723.

Allesina, S., Alonso, D., Pascual, M., 2008. A general model for food web structure. Science 320, 658-661.

Bascompte, J., Melián, C.J., 2005. Simple trophic modules for complex food webs. Ecology 86, 2868-2873.

Bascompte, J., Melián, C.J., Sala, E., 2005. Interaction strength combinations and the overfishing of a marine food web. In: Proceedings of the National Academy of Sciences, USA, vol. 102, pp. 5443-5447.

Brose, U., Cushing, L., Berlow, E.L., Jonsson, T., Banasek-Richter, C., Bersier, L.-F., Blanchard, J.L., Brey, T., Carpenter, S.R., Cattin Blandenier, M.-F., Cohen, J.E., Dawah, H.A., Dell, T., Edwards, F., Harper-Smith, S., Jacob, U., Knapp, R.A., Ledger, M.E., Memmott, J., Mintenbeck, K., Pinnegar, J.K., Rall, B.C., Rayner, T., Ruess, L., Ulrich, W., Warren, P.H., Williams, R.J., Woodward, G., Yodzis, P., Martinez, N.D., 2005. Body sizes of consumers and their resources. Ecology $86,2545$.

Brose, U., Jonsson, T., Berlow, E.L., Warren, P., Banasek-Richter, C., Bersier, L.F. Blanchard, J.L., Brey, T., Carpenter, S.R., Blandenier, M.F., Cushing, L., Dawah, H. A., Dell, T., Edwards, F., Harper-Smith, S., Jacob, U., Ledger, M.E., Martinez, N.D., Memmott, J., Mintenbeck, K., Pinnegar, J.K., Rall, B.C., Rayner, T.S., Reuman, D.C., Ruess, L., Ulrich, W., Williams, R.J., Woodward, G., Cohen, J.E., 2006. Consumerresource body-size relationships in natural food webs. Ecology 87, 2411-2417.

Camacho, J., Guimerà, R., Amaral, L.A.N., 2002. Robust patterns in food web structure. Phys. Rev. Lett. 88, Art. no. 228102-4.

Camacho, J., Arenas, A., 2005. Universal scaling in food-web structure. Nature 435, E3-E4.

Camacho, J., Stouffer, D.B., Amaral, L.A.N., 2007. Quantitative analysis of the local structure of food webs. J. Theor. Biol. 246, 260-268.

Capitán, J.A., Cuesta, J.A., Bascompte, J., 2009. Statistical mechanics of ecosystem assembly. Phys. Rev. Lett. 103, Art. no. 168101.

Capitán, J.A., Cuesta, J.A., 2010. Catastrophic regime shifts in model ecological communities are true phase transitions. J. Stat. Mech. Art. no. P10003. doi: http://dx.doi.org/10.1088/1742-5468/2010/10/P10003, in press.

Capitán, J.A., Cuesta, J.A., 2011. Species assembly in model ecosystems. I. Analysis of the population model and the invasion dynamics. J. Theor. Biol. 269, 330-343.

Capitán, J.A., Cuesta, J.A., Bascompte, J., 2011. Species assembly in model ecosystems. II. Results of the assembly process. J. Theor. Biol. 269, 344-355.

Cattin Blandenier, M.-F., 2004. Food Web Ecology: Models and Application to Conservation. Ph.D. Thesis.

Cattin, M.-F., Bersier, L.-F., Banašek-Richter, C., Baltensperger, R., Gabriel, J.-P., 2004. Phylogenetic constraints and adaptation explain food-web structure. Nature 427, 835-839.

Cohen, J.E., 1977. Food webs and the dimensionality of trophic niche space. In: Proceedings of the National Academy of Sciences, USA, vol. 74, pp. 4533-4536.

Cohen, J.E., Newman, C.M., 1985. A stochastic theory of community food webs. I. Models and aggregated data. Proc. R. Soc. B 224, 421-448.

Cohen, J.E., 1989. Ecologists Co-operative Web Bank (ECOWeBTM). Version 1.0. Machine Readable Data Base of Food Webs. Rockefeller University, NY.

Cohen, J.E., Briand, F. Newman, C.M., 1990. Community Food Webs: Data

and Theory. Springer-Verlag, Berlin.

Cohen, J.E., Schittlerb, D.N., Raffaelli, D.G., Reuman, D.C., 2009. Food webs are more than the sum of their tritrophic parts. In: Proceedings of the National Academy of Sciences, USA, vol. 106, pp. 22335-22340.

Dawah, H.A., Hawkins, B., Claridge, M., 1995. Structure of the parasitoid communities of grass-feeding chalcid wasps. J. Anim. Ecol. 64, 708-720.

Dunne, J.A., Williams, R.J., Martínez, N.D., 2002. Food-web structure and network theory: the role of connectance and size. In: Proceedings of the National Academy of Sciences, USA, vol. 99, pp. 12917-12922.

Eklöf, A., Jacob, U., Kopp, J., Bosch, J., Castro-Urgal, R., Chacoff, N.P., Dalsgaard, B., de Sassi, C., Galetti, M., Guimarães, P.R., Lomáscolo, S.B., Martín González, A.M., 
Pizo, P., Rader, R., Rodrigo, A., Tylianakis, J.M., Vázquez, D.P., Allesina, S., 2013. The dimensionality of ecological networks. Ecol. Lett. 16, 577-583.

Guimerà, R., Stouffer, D.B., Sales-Pardo, M., Leicht, E.A., Newman, M.E.J., Amaral, L.A.N. 2010. Origin of compartmentalization in food webs. Ecology 91, 2941-2951.

Harper-Smith, S., Berlow, E.L., Knapp, R., Williams, R.J., Martínez, N.D., 2005. Communicating ecology through food webs: visualizing and quantifying the effects of stocking alpine lakes with trout. In: de Ruiter, P., Wolters, V., Moore, J. (Eds.), Dynamic Food Webs. Multispecies Assemblages, Ecosystem Development and Environmental Change. Academic Press, Burlington, pp. 407-423.

Hall, S.J., Raffaelli, D., 1991. Static patterns in food webs: lessons from a large web. J. Anim. Ecol. 60, 823-842.

Hutchinson, G., 1957. Concluding remarks. Cold Springs Harbor Symp. Quant. Biol. $22,415-427$.

Jennings, S., Pinnegar, J.K., Polunin, N.V.C., Warr, K.J., 2002. Linking size-based and trophic analyses of benthic community structure. Mar. Ecol. Prog. Ser. 226 $77-85$

Jonsson, T., 1998. Food Webs and the Distribution of Body Sizes. Ph.D. Thesis.

Kendall, B., Briggs, C., Murdoch, W., Turchin, P., Ellner, S., McCauley, E., Nisbet, R., Wood, S., 1999. Why do populations cycle? A synthesis of statistical and mechanistic modeling approaches. Ecology 80, 1789-1805.

Lawton, J.H., Warren, P.H., 1988. Static and dynamic explanations for patterns in food webs. Trends Ecol. Evol. 3, 242-245.

Lafferty, K., Dobson, A.P., Kuris, A., 2006. Parasites dominate food web links. In: Proceedings of the National Academy of Sciences, USA, vol. 103, pp. 1121111216.

Levine, S., 1980. Several measures of trophic structure applicable to complex food webs. J. Theor. Biol. 83, 195-207.

Lewis, H.M., Law, R., 2007. Effects of dynamics on ecological networks. J. Theor. Biol. 247, 64-76.

Loeuille, N., Loreau, M., 2005. Evolutionary emergence of size-structured food webs. In: Proceedings of the National Academy of Sciences, USA, vol. 102, pp. 57615766 .

Martínez, N.D., 1991. Artifacts or attributes? Effects of resolution on the Little Rock Lake food web. Ecol. Monogr. 61, 367-392.

Memmott, J., Martínez, N.D., Cohen, J.E., 2000. Predators, parasitoids and pathogens: species richness, trophic generality and body sizes in a natural food web. J. Anim. Ecol. 69, 1-15.

Opitz, S., 1996. Trophic Interactions in Caribbean Coral Reefs. ICLARM Technical Report 43, Manila, Philippines.

Pascual, M., Dunne, J.A. (Eds.), 2006. Ecological Networks: Linking Structure to Dynamics in Food Webs. Oxford University Press, Oxford, UK.

Petchey, O.L., Beckerman, A.P., Riede, J.O., Warren, P.H., 2008. Size, foraging, and food web structure. In: Proceedings of the National Academy of Sciences USA, vol. 105, pp. 4191-4196.
Polis, G.A., 1991. Complex trophic interactions in deserts: an empirical critique of food-web theory. Am. Nat. 138, 123-155.

Reuman, D.C., Desharnais, R.A., Costantino, R.F., Ahmad, O.S., Cohen, J.E., 2006 Power spectra reveal the influence of stochasticity on nonlinear population dynamics. In: Proceedings of the National Academy of Sciences USA, vol. 103 pp. 18860-18865.

Rezende, E.L., Albert, E.M., Fortuna, M.A., Bascompte, J., 2009. Compartments in a marine food web associated with phylogeny, body mass, and habitat structure. Ecol. Lett. 12, 779-788.

Rossberg, A.G., Brännström, A., Dieckmann, U., 2010. Food-web structure in lowand high-dimensional trophic niche spaces. J. R. Soc. Interface 7, 1735-1743.

Schoener, T.W., 1989. Food webs from the small to the large. Ecology 79, 2013-2018.

Stouffer, D.B., Camacho, J., Guimerà, R., Ng, C.A., Amaral, L.A.N., 2005. Quantitative patterns in the structure of model and empirical food webs. Ecology 86, 1301-1311.

Stouffer, D.B., Camacho, J., Amaral, L.A.N., 2006. A robust measure of food web intervality. In: Proceedings of the National Academy of Sciences USA, vol. 103, pp. 19015-19020.

Stouffer, D.B., Camacho, J., Jiang, W., Amaral, L.A.N., 2007. Evidence for the existence of a robust pattern of prey selection in food webs. Proc. R. Soc. B 274, 1931-1940.

Stouffer, D.B., Ng, C.A., Amaral, L.A.N., 2008. Ecological engineering and sustainability: a new opportunity for chemical engineers. AIChE J. 54, 3040-3047.

Stouffer, D.B., Rezende, E.L., Amaral, L.A.N., 2011. The role of body mass in diet contiguity and food-web structure. J. Anim. Ecol. 80, 632-639.

Stouffer, D.B., Bascompte, J., 2011. Compartmentalization increases food web persistence. In: Proceedings of the National Academy of Sciences USA, vol. 108, pp. 3648-3652.

Stouffer, D.B., Sales-Pardo, M., Sirer, M.I., Bascompte, J., 2012. Evolutionary conservation of species' roles in food webs. Science 335, 1489-1492.

Warren, P.H., 1989. Spatial and temporal variation in a freshwater food web. Oikos 55, 299-311.

Williams, R.J., Martínez, N.D., 2000. Simple rules yield complex food webs. Nature 404, 180-183.

Williams, R.J., Martínez, N.D., 2004. Limits to trophic levels and omnivory in complex food webs: theory and data. Am. Nat. 163, 458-468.

Woodward, G., Ebenman, B., Emmerson, M., Montoya, J.M., Olesen, J.M., Valido, A Warren, P.H., 2005. Body size in ecological networks. Trends Ecol. Evol. 20, 402-409.

Yodzis, P., 1998. Local trophodynamics and the interaction of marine mammals and fisheries in the Benguela ecosystem. J. Anim. Ecol. 67, 635-658.

Zook, A.E., Eklöf, A., Jacob, U., Allesina, S., 2011. Food webs: ordering species according to body size yields high degree of intervality. J. Theor. Biol. 271 106-113. 